\title{
Affectionate Touch in the Context of Breastfeeding and Maternal Depression Influences Infant Neurodevelopmental and Temperamental Substrates
}

\author{
Jillian S. Hardin Nancy Aaron Jones Krystal D. Mize Melannie Platt \\ Charles E. Schmidt College Science Department of Psychology, Florida Atlantic University, Jupiter, FL, USA
}

\section{Keywords}

Touch · Electrophysiology · Feeding · Temperament · Early experience $\cdot$ Depression

\begin{abstract}
Background: While numerous studies have demonstrated maternal depression's influence on infant brain development, few studies have examined the changes that occur as a consequence of co-occurring experiential factors that affect quality of mother and infant affectionate touch as well as infant temperament and neurophysiological systems. The aim of the study was to examine the interactive effects of maternal depression and breastfeeding on mother and infant affectionate touch and infant temperament and cortical maturation patterns across early development. Methods: 113 mothers and their infants participated when infants were 1 and 3 months of age. Questionnaires to assess maternal depressive symptoms, feeding, and temperament were completed. Tonic EEG patterns (asymmetry and left and right activity) were collected and the dyads were video-recorded during feeding to assess mother and infant affectionate touch patterns. Results: Data analysis showed that EEG activity and mother-infant affectionate touch differed as a
\end{abstract}

karger@karger.com

(c) 2021 S. Karger AG, Basel

www.karger.com/nps

Karger" function of mood and feeding method. Notably, only infants of depressed mothers that bottle-fed showed right frontal EEG asymmetry and attenuated change in the left frontal region across 3 months. Breastfeeding positively impacted affectionate touch behaviors and was associated with increased left and decreased right frontal EEG activation even for depressed groups. Furthermore, a model incorporating physiology, maternal depression, touch, temperament, and feeding indicated significant prediction for infant affectionate touch (with breastfeeding and affectively positive temperament demonstrating the strongest prediction). Conclusion: The findings suggest that breastfeeding and the infant's positive temperament influence mother-infant affectionate touch patterns and result in neuroprotective outcomes for infants, even those exposed to maternal depression within early development. @ $2021 \mathrm{~S}$. Karger AG, Basel

\section{Introduction}

Previous research has shown that maternal depression negatively impacts the social interactions between mothers and infants affects temperamental emotionality in 
combination with the physiological development of infants $[1-3]$. As depressed mothers are less likely to provide soothing touch, less able to detect changes in facial expressions, and more likely to have trouble regulating their own emotions, infants of depressed mothers are at a high risk of atypical and potentially dysregulated interaction. In addition to behavioral markers, infants of depressed mothers exhibit similar brain functioning patterns as their depressed mothers [4-6], and these patterns are linked to temperament characteristics as well [3]. Therefore, contemporary research studies focused on uncovering risk factors associated with maternal depression should entail an examination of experiential factors that may influence mother-infant interactions and the development of infant neurophysiology and temperament. The current study focuses on dyadic affectionate tactile patterns during feeding for 2 reasons: (1) touch is a form of mutual interaction established in early infancy, used to communicate needs, soothe, and downregulate stress responses, and (2) dyads spend a significant amount of time feeding across the first 3 months postpartum. The current study specifically explores affectionate touch, defined as light and deep touch (i.e., stroking, massaging, and caressing, but see Materials and Methods for a detailed overview) initiated by either mother (mother affectionate touch) or infant (infant affectionate touch). Our aim is to investigate the interactive effects of the feeding environment and maternal depression (co-occurring experiential factors) during infancy as potentially affecting quality of dyadic (patterns of mother and infant affectionate touch), infant temperament, and the corresponding maturation of the infant brain, specifically focusing on alterations in EEG activation patterns across development.

\section{Maternal Depression's Effect on Infants}

Conservative estimates suggest that maternal depression affects about 1 in 9 mothers [7], potentially influencing not only maternal interactions with the infant but also impacting infant development and the dyads' socio-emotional patterns during pivotal points in development [4, 8 , 9]. Depressed mothers are more likely to report their infants temperament as being difficult [10] and infants of depressed mothers show disruptions in the development of emotional regulation, including altered patterns of social interactions [11].

Maternal depression effects are present in infancy and extend through childhood and potentially into adulthood. Maternal depression increases risk for childhood behavioral problems [12] and, across the lifespan, children of depressed mothers are more likely to develop de- pression themselves and at earlier ages than children of nondepressed mothers [13]. Negative affect early in life is known to continue into later infancy [14], making early infancy a crucial time to examine the initiation of the mother-infant relationship within its larger environmental context.

\section{Theory Underlying Research: Experiences in Infant Development}

Two theories guided the current study. First, Greenough's model of neural plasticity includes a specific form of neural plasticity called "experience-dependent" plasticity, in which early brain development is altered by unique individual experiences [15]. According to this framework, environmental interactions such as interpersonal exchanges alter development [16]. In addition, the biopsychosocial model acknowledges neural plasticity in early development, but also contends that it is necessary to study interactive biological, psychological, and social processes as experiences that impact individual differences in development [17]. Thus, it is not surprising that researchers find infant and child EEG patterns are associated with differences in the child-rearing environment. For instance, positive maternal affect is associated with maturational changes in infant EEG power across the first 24 months of life [18]. In infants of depressed mothers, researchers found that right frontal EEG asymmetry patterns (a dysregulated physiological pattern linked to depressive disorders) in 1-month-olds were reduced during and after a 10-min infant massage [19]. In addition, more positive dyadic interaction and left frontal EEG asymmetry (a more regulated EEG pattern) has been associated with infants of depressed mothers who have a stable breastfeeding relationship with their mothers [3].

Second, emotional dispositions are core components of the structure of temperament, and individual differences in emotionality emerge and are related to neurophysiological and neurobiological functioning from very early childhood [20-22]. For instance, in the current study, hemispheric lateralization, specifically patterns of right and left frontal EEG asymmetry, may reflect neurobiological dispositions for both temperamental characteristics and risk for depression. Contemporary descriptions of temperament [23] acknowledge that it is a neurobiological process regulated, in part, through the same neurobehavioral systems underlying mental illness, such as depression. The Functional Ensemble of Temperament (FET) model [24] posits that dimensions of temperament are regulated by monoamines such as noradrenaline and other hormones and opioid receptors. In- 
terestingly, noradrenaline has a higher density of receptors in the right hemisphere of the brain (and is also known to downregulate prolactin, a hormone largely involved in breastfeeding) [25]. Oxytocin, released in response to interpersonal touch and breastfeeding, appears to decrease noradrenergic response [26]. Thus, hormonal activity underlying EEG asymmetry, infant feeding, affectionate dyadic touch, temperament, and depressive symptoms appear to lie within, as Sulis [23] postulates, one neurobiological continuum, which likely impacts infant brain plasticity.

Taken together, these theories suggest that early experiences likely influence temperamental arousal and regulation underlying EEG patterns in infancy and childhood. This is important given what we know about brain plasticity in infancy as early contextual factors, such as maternal depression and feeding method, may cumulatively alter mother-infant relationship outcomes as well as neurophysiological functioning across development.

\section{Infant Frontal EEG Asymmetry and Power}

During childhood and across adulthood, patterns of frontal EEG asymmetry reflect stable predispositions for either greater relative right frontal asymmetry or left frontal asymmetry [27]. Fox and his colleagues [20, 28] have reported on a series of studies that demonstrate EEG patterns remain temporally stable. Greater left frontal asymmetry is generally accepted as a marker for overall brain maturation [29], with a shift from right to left in infancy coinciding with normative patterns of development [30].

Asymmetry patterns in certain infant populations, such those of depressed mothers, however, differ from the asymmetry patterns of typically developing infants and children $[3,31]$. While EEG asymmetry measures the balance of the right and left hemisphere activity, it is documented that infants of depressed mothers exhibit patterns of right frontal asymmetry, due, in part, to hypoactivation of the left hemisphere within the frontal region [32]. This pattern of brain activation, that is, greater right asymmetry due to left frontal hypoactivation, is similar to the pattern observed in depressed adults $[33,34]$ and is thought to represent heightened negative affect as well as motor tendencies for withdrawal and inhibited approach behaviors [22, 35].

While some studies have produced inconclusive outcomes with infants [36], a meta-analysis reported that infants of depressed mothers not only interacted less with their mothers, but the dyads exhibited consistent patterns of right frontal asymmetry that were also linked to nega- tive emotions and emotional dysregulation [37]. The predisposition for infants of depressed mothers to display lateralized patterns of EEG asymmetry distinct from those of nondepressed groups has been documented in infants from the newborn period to 12 months [38-40]. Early examination of the experiential factors and their $\mathrm{cu}-$ mulative effects on physiological and temperamental dispositions that manifest in behavioral interactions with parents is important, as it provides insight into the differential influences that both risk factors and protective factors may exert within specific environmental contexts.

\section{Importance of Touch during Infancy and the Study of} Affectionate Dyadic Touch

In typically developing infants, affectionate touch between mother and infant has been reliably demonstrated as a predictor of synchronous mother-infant interaction [41], facilitating the dyadic relationship and emotional connection $[42,43]$. Maternal touch also has a downregulating effect on the infant stress response system, even in the absence of maternal vocalization and gaze [44]. For example, when maternal touch is added during the stressinvoking still-face procedure, infants show less reactive facial expressions [45]. Furthermore, maternal touch not only protects the infant from psychological stressors but also from negative physical experiences. Other researchers have demonstrated that tactile stimulation during infancy and childhood enhances cortical activation through sensory and social input [46-48]. Collectively, the research suggests that touch plays an important role in healthy infant socio-emotional development.

In early infancy, dyadic touch serves as an important form of interaction between mother and infant but maternal depression impacts the touch patterns of mothers and infants. For example, previous research has demonstrated that infants of depressed mothers are less soothed by maternal touch in comparison to infants of nondepressed mothers $[49,50]$. The infants' reactions to their depressed mothers may in part be explained by maternal unavailability in the form of low amounts of nurturing touch [51], as depressed mothers touch their infants less or in more intrusive and rough manner than nondepressed mothers [52]. One goal of the current study is to examine whether dyadic affectionate touch adaptively (or maladaptively) influences mother-infant outcomes and infant temperament in different groups. Specifically, the development of mother-directed infant and infant-directed maternal affectionate touch patterns will be probed in the present study not only in the context of maternal depression but also in consideration of different patterns 
of mother-infant feeding. Additionally, the association between affectionate touch and temperamental reactivity and regulation of the infant will be examined.

\section{Feeding Interactions as a Social Context}

Given that feeding is a necessary part of infant caregiving, it is one of the earliest contexts for mother-infant social interactions. Previous studies have also found differences in touch patterns of breastfeeding mothers and their infants. An early study found that breastfeeding dyads engaged in higher levels of mutual touch than bottlefeeding dyads [53], and more contemporary researchers concluded that breastfeeding mothers spent more time stroking their infants than bottle-feeding mothers [54]. The effects of breastfeeding on the mother-infant relationship appear to persist through childhood and adolescence, with breastfeeding predicting greater maternal sensitivity over a 10-year period [55]. Importantly, depressed mothers who breastfed also displayed less intrusive behaviors and higher levels of touch and perceived their infants as less irritable $[3,54,56]$.

\section{The Current Study}

Mother-infant affectionate tactile interactions, infant temperament ratings, and infant EEG asymmetry and power were examined utilizing a quasi-experimental design in a sample of depressed and nondepressed, breastand bottle-feeding dyads during feeding sessions at 1 and 3 months postpartum. Past research has addressed touch patterns in depressed and nondepressed mother-infant dyads [11, 57]; yet, no research has been conducted on affectionate tactile behaviors of both mother and infant during feeding sessions coupled with the examination of interactive effects of the infant feeding environment and maternal depressive mood on infant EEG patterns and temperamental characteristics.

Two hypotheses were tested. First, we expected that mother and infant affectionate touch would be enhanced for breastfeeding dyads. Specifically, because breastfeeding has been shown to facilitate mother and infant interactive touch even in the presence of maternal depression [3], it was expected that the depressed mothers who breastfeed their infants (M-BFD) as well as the infants themselves (I-BFD) would exhibit higher levels of affectionate touch patterns at 1 and 3 months as compared to depressed mothers (M-FFD) and their infants that bottlefeed (I-FFD). Associations between mother and infant affectionate touch and temperamental qualities were also examined as well as whether infants of depressed mothers who bottle-feed or breastfeed exhibit variation in mater- nal-reported temperamental characteristics. Second, based on previous studies showing an association between maternal depression and infant EEG patterns, here we wanted to examine whether feeding and maternal depression are interactively related to changes in resting frontal EEG asymmetry and power. It was expected that (a) I-BFD would show more left frontal EEG asymmetry patterns than I-FFD, who were expected to show increases in relative right frontal EEG asymmetry across development, and (b) I-BFD would show increases in left frontal region activation (less power) across age.

We also conducted an exploratory analysis to examine infant affectionate touch patterns. We examined the factors that predicted 3-month mother-directed affectionate infant touch by exploring the role of breastfeeding duration, maternal depression status, frontal region EEG asymmetry, positive temperament, and infant touch at earlier periods of development.

\section{Materials and Methods}

\section{Participants}

A total of 113 mother-infant dyads participated at the 1-month laboratory visit and $81(72 \%)$ mother-infant dyads returned at the 3 -month visit. The sample consisted of predominantly middleand upper middle-class $(82.1 \%)$, well-educated (97\% had a high school education or beyond), married (92.9\%) mothers ( $M$ maternal age $=31.00$ years; $\mathrm{SD}=4.74)$. Infants were brought to the laboratory at 1 month of age $(\mathrm{M}=37.14$ days; $\mathrm{SD}=5.88)$ and returned for a second visit at 3 months of age $(M=95.36$ days; $S D=11.39)$. For more information about the general maternal characteristics of the sample at each age, see Table 1. Infants were all full term ( $>37$ weeks) with no significant developmental delays (confirmed by maternal interview). Fifty-five percent of the infants were female and $45 \%$ were male. There were no differences between groups for these demographic variables, including maternal demographics and infant birth characteristics $(p s>0.05)$. The study was approved by the Florida Atlantic University Institutional Review Board, and mothers signed consents for both their own participation and their infants' participation in the study.

\section{Mother-Infant Dyad Group Classification}

Mother-infant dyads were assigned to 1 of 4 different groups based on the mother's depression status and primary feeding method across the first 3 months postpartum (Table 1) in order to examine the interactive effects of these variables in the analyses: a depressed mother group that breastfed (M-BFD), a nondepressed group that breastfed (M-BFND), a depressed group that either bottle-fed formula or did mixed feedings (M-FFD), and a nondepressed group that bottle-fed or did mixed feedings (M-FFND).

Depression Classification

The Center for Epidemiological Studies Depression Scale (CES-D), a 20-item questionnaire shown to be a reliable measure of depression [58], was used to screen mothers for depressive 
symptoms. The CES-D scores range from 0 to 60 . A score of 12 or below is defined as "nondepressed" and a score of 16 or above is defined as "depressed." The mothers assigned to either of the 2 depressed groups (depressed and breastfeeding or depressed and bottle-feeding) scored above 16 on the CES-D during the 1-month visit (Table 1). Maternal reports of depressive symptoms were higher during the 1-month visit, remained higher than the nondepressed group across the study's duration, and both groups decreased in depressive symptoms across time. Depressed and nondepressed groups differed in depressive symptoms at both ages, $t$ between 5.65 and $14.38, p=0.002$, and scores were significantly correlated across time $(r(79)=0.48, p<0.001)$.

\section{Feeding Status Classification}

Assignment of the feeding groups was dependent on motherreported feeding practices and the feeding method used during the laboratory visits. Mothers in the breastfeeding group were predominately breastfeeding (Table 1) and no formula was given to the infant at the 1- and 3-month visits. Mothers who bottle-fed exclusively, used predominantly formula or both feeding methods, and used formula during the visits were assigned to the bottlefeeding group. Although the operational definition of the breastfeeding group for this study is a conservative approach to assigning the "breastfeeding" classification, it was done in an attempt to study the behavioral experiences of breastfeeding and its association with the social environment rather than the nutritive benefits of breastfeeding versus formula feeding [59].

Mothers were also queried on the type of food they fed their infants across the first 3 months. We recorded all the foods ingested and that the mother reported giving her infant and when she stopped using any breast milk by indicating the number of days the mother breastfed across the first 3 months. The average length of breastfeeding was 59 days $(\mathrm{SD}=36.6$; range $=0-98$ days $)$ (Table 1$)$.

\section{Infant Temperament: Infant Behavior Questionnaire}

Infant temperament was reported by mothers using the Infant Behavior Questionnaire-Revised (IBQ-R) [21]. The IBQ-R is a 191-item questionnaire, which measures maternal perceptions of 14 dimensions of infant temperament including infant activity level, pleasure, arousal, fear, sadness approach, vocal reactivity, duration of orienting, smiling and laughter, high-intensity pleasure, low-intensity pleasure, soothability, falling reactivity/rate of recovery from distress, perceptual sensitivity, sadness, approach, vocal reactivity, and cuddliness.

\section{Procedures}

Mothers and infants were assessed at our developmental laboratory at each age. Mothers completed questionnaires (including family demographics, self-reported feeding, depression questionnaires, and the Infant Behavior Questionnaire [IBQ]; [21]) at each age. Mother-infant dyads participated in play and feeding sessions, and a 5-min infant baseline EEG was recorded at each age. The order of tasks was randomized based on the infant's needs during the visit. All physiological and behavioral assessments were recorded and saved for later coding and analyses.

Behavioral Coding of Mother and Infant Touch Behaviors

The feeding session was recorded, with cameras focused on both the mother and infant, for as long as it took the mother to feed her infant. However, only the first $5 \mathrm{~min}$ of the recording was used
Table 1. Demographic characteristics of the families

\begin{tabular}{lcc}
\hline & 1 month & 3 months \\
\hline Race/ethnicity (N) & & \\
Caucasian & $97(85.8 \%)$ & $70(86.4 \%)$ \\
African-American & $9(8.0 \%)$ & $5(6.2 \%)$ \\
Hispanic & $2(1.8 \%)$ & $2(2.5 \%)$ \\
More than 1 group & $5(4.4 \%)$ & $4(4.9 \%)$ \\
\hline Marital status $(N)$ & & \\
Married & $106(93.8 \%)$ & $78(96.4 \%)$ \\
Engaged & $2(1.8 \%)$ & $1(1.2 \%)$ \\
Single & $5(4.4 \%)$ & $2(2.5 \%)$ \\
\hline Employment $(N)$ & & \\
Outside of home & $50(44.2 \%)$ & $32(39.5 \%)$ \\
In school & $4(3.5 \%)$ & $2(2.5 \%)$ \\
Full-time mother & $59(52.2 \%)$ & $47(58.0 \%)$ \\
\hline Parity $(N)$ & & \\
One child & $43(38.1 \%)$ & $26(32.1 \%)$ \\
Two children & $44(38.9 \%)$ & $35(43.2 \%)$ \\
More than 2 children & $26(23.0 \%)$ & $20(25.7 \%)$
\end{tabular}

Depression and feeding group $(N)$

Depressed and breastfeeding $\quad 14 \quad 9$

Depressed and bottle-feeding $\quad 14 \quad 8$

Nondepressed and breastfeeding $\quad 59 \quad 49$

Nondepressed and bottle-feeding $\quad 26 \quad 15$

CES-D scores (mean [SD])

Depressed group $\quad 21.5(6.60) \quad 9.56(5.74)$

Nondepressed group $\quad 6.57(4.10) \quad 4.48(3.73)$

Days breastfeeding (mean [SD])

Stable breastfeeding group

$87.15(11.56)$

Bottle or mixed feeding group

$30.60(30.69)$

CES-D, Center for Epidemiological Studies Depression Scale.

for behavioral coding analyses. Once the mothers began the feeding session, they were left alone to breastfeed or bottle-feed their infants.

The current study utilized a modified version of the Touch Scoring Instrument [60] to classify maternal touch patterns. In this coding system, touching behaviors are scored on a second-by-second (using Observer coding program) basis on 7 categories of touch. The 7 categories are as follows: 7 - firm touch: firm patting, stroking, or massaging with the whole hand; 6 - light active touch: affectionate kissing, caressing, or stroking; 5 - passive touch: passive contact, resting the hand in contact with the infant, or comforting presence for infant without interrupting flow of attention, has been shown as a component of the affectionate composite; 4 - not affectionate/passive/reactive: any form of maternal touch that does not fall into the affectionate, passive, or reactive touch categories; 3 - no touch; 2 - awkward holding: holding the child in an uncomfortable manner with an uninterested or neglectful attitude; and 1 - rough handling: exercising forceful or abrupt re- 
straint or physical control of the child with an angry or punitive quality.

A variation of the infant touch scale developed by Moszkowski and Stack [61] was used to code infant touch behaviors on a second-by-second basis using Observer. To be classified as infant touch, the infant had to actively initiate or reciprocate touch with the mother. Seven types of touch were coded: 7 - stroke (rub, caress, wipe): lateral movement of the hand; 6 - finger (manipulate): running the tip of the fingers over a surface; 5 - static: hand remains still while in contact with mother; 4 - no touch: hand not in contact with anything; 3 - pat: up and down motion of the hand against a surface; 2 - pull (push, lift): raising/lowering of a stimulus or exerting pressure against an object; and 1 - grab (clutch, clasp): curling of fingers around a stimulus, active grab. Touch was also examined by grouping touch in 3 categories: self, mother, and other (defined as a location the infant touches other than the mother or self).

Camera equipment malfunctions, missing data due to infant sleeping/fussiness, and attrition at 3 months yielded unusable mother-infant touch data for 22 mother-infant dyads at the 1 -month laboratory visit and 5 mother-infant dyads at the 3 -month laboratory visit. Usable data were collected for 91 mother-infant dyads at the 1-month laboratory visit and 76 mother-infant dyads at the 3-month laboratory visit. Twenty percent of the videos were coded by two or more coders to establish reliability. Cohen kappa scores for the reliability of the touch scale coding ranged from 0.85 to 0.95 and were checked periodically across the time data coding was performed.

Composite Scores for Affectionate Touch: Mother and Infant

Affectionate touching behaviors were analyzed using percentages (derived from duration in touch states during the 5-min feeding sampling) at the 1-and 3-month visits. A composite score from the quality scores was created for maternal active affectionate touch (AT-M) for both the 1- and 3-month visits. Maternal deep affectionate touch (firm patting, stroking, massaging with the whole hand) and maternal light affectionate touch (affectionate kissing, caressing, or stroking, grazing, hugging) were combined into a single variable, as these forms of touch are considered dimensions of affectionate touch [41].

For the infant touch coding, a composite score was created to reflect infant affectionate tactile behaviors (AT-I) for the 1- and 3-month visits. Infant deep touch (stroking, rubbing, caressing, and wiping), infant light touch (running the tip of the fingers on the mother), and infant touch (resting hand on mother) were used to create the infant affectionate touch variable. Only tactile behaviors directed toward the mother were classified as infant affectionate touch.

\section{Electroencephalograph Recordings}

Infant baseline EEG recordings were collected while infants were in a quiet awake state and recordings lasted for $5 \mathrm{~min}$. A stretch Lycra cap (Electro Cap, Inc.) with the international 10-20 system was used to measure the EEG recordings. Impedances were brought below $5 \mathrm{~K}$ ohms. Omni prep abrasive gel and electrode gel were placed in the 8 electrode sites measured. The sites utilized were as follows: mid frontal (F3 and F4), central (C3 and C4), parietal (P3 and $\mathrm{P} 4)$, and occipital (O1 and $\mathrm{O} 2)$. All 8 sites were referenced to the vertex $(\mathrm{Cz})$ during data collection. While there is no consensus on the most appropriate reference site in infancy, linked mastoids, the vertex $(\mathrm{Cz})$ and average reference have been utilized in the past, however, we choose to use the vertex reference to be consistent with other studies in the area $[2,3]$. EOG was also obtained to easily remove eye movement artifacts from the EEG data. Two mini electrodes were placed on the infant's face, one on the outer canthus and the other on the supra orbit position. The EEG electrical signal was amplified using SA Instrumentation Bioamps and bandpassed from 1 to $100 \mathrm{~Hz}$. The EEG activity from each lead was streamed onto a computer screen. The EEG sampling rate online rate was 512 samples per second. The EEG recordings were saved to a computer hard drive using Snapstream v. 3.21 from HEM Data Corp. As is standard for the software from James Long Co. (Caroga Lake, NY, USA), the data were analyzed using a discrete Fast Fourier transform using a Hanning window with a $50 \%$ overlap. A total of 921 -month-old infants had usable data in all regions and 73 3-month-old infants had usable data in all regions. In total, 66 infants had usable data at both 1- and 3-month data in all regions. On average, infants with usable data had over 2 min of artifact-free data to analyze at each age $(M=123.36 \mathrm{~s}$ at 1 month, $\mathrm{SD}=77.63$; and $\mathrm{M}=130.31 \mathrm{~s}$ at 3 months, $\mathrm{SD}=85.44$ ).

Although there is no universally accepted criterion for frequency bands used for infants, researchers [2] have demonstrated a shift in power using the $3-6 \mathrm{~Hz}$ frequency bands in young infants and the $6-9 \mathrm{~Hz}$ range in older infants $[4,62]$. In this data set, we also examined the spectral plots at each age and the activity clustered around the $3-6 \mathrm{~Hz}$ band and $6-9 \mathrm{~Hz}$ at 1 and 3 months, respectively. Commonly, the literature surrounding EEG frequency relates a clustering of activity as indicative of the alpha band activity, with lower alpha power values related to higher activation patterns within a frequency band [63].

Natural log conversions were used on all 1- and 3-month EEG recordings. The log transformed data were used consistently across all power and asymmetry analyses. The asymmetry scores were computed $(\ln [$ right $]-\ln [$ left $])$, so that negative scores reflect relative right hemispheric activity and positive scores reflect relative left hemispheric activity. Data collection and analysis procedures of the 3-month visit were identical to those of the 1-month visit. To check on the reliability of the EEG data, bivariate correlations between nearby sites were examined within age. All correlations were significant $(p<0.05)$ with $r$ 's ranging from 0.986 to 0.746 within regions and from 0.891 to 0.259 across nearby regions.

\section{Data Analytic Strategy}

Touch pattern data (for the mother and the infant) were analyzed with 1-way ANOVAs using the 4 group classifications (MBFD, M-FFD, M-BFND, M-FFND) as the fixed factor. ANOVAs were conducted on 4 groups (rather than depressed and feeding groups separately) in order to examine potential overall interactive effects of infant feeding method and maternal depression. Followup tests were conducted to examine specific group differences using Tukey HSD and/or 1-tailed $t$ tests if the ANOVA was significant. One-tailed $t$ tests were conducted because of the small sample size in the depressed group and the specific, directional hypotheses for the depressed groups. All effect sizes are reported using Hedges' $g$ due to the unequal sample sizes.

To examine the relationship between temperament and touch, correlations between mother-rated IBQ scores and affectionate touch of mothers and infants scores were conducted. In addition, we used repeated measures multivariate analyses to examine whether temperament ratings differed for each group, examining 
Table 2. Mother and infant touch behavior percentages at 1 and 3 months

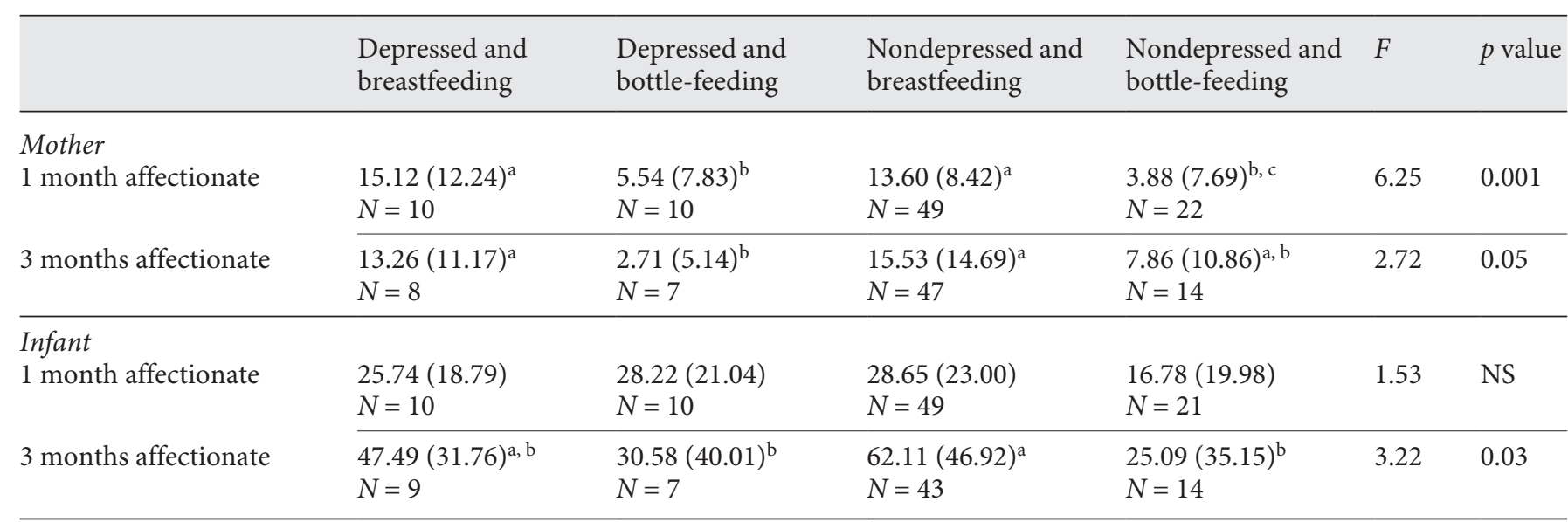

Percentages reflect the mean percentage of touch behaviors observed during a 5-min feeding sampling. Standard deviations appear in parentheses. Different letter superscripts in each row are significantly different from each other.

both 4 group classifications for all 6 subscales of the temperament questionnaire.

For EEG analyses, repeated measures MANOVAs were conducted to examine asymmetry scores for overall group (M-BFD, M-FFD, M-BFND, M-FFND), region (frontal, central, parietal occipital), and age (1 and 3 months) effects. Follow-up analyses were conducted for each region and/or each age if omnibus tests yielded significant multivariate outcomes. Power scores were then analyzed as a follow-up to asymmetry analyses in order to glean further information regarding hemispheric (left or right) activation. While EEG asymmetry scores provide a measure of relative hemispheric activity, EEG power can be examined separately to provide information regarding hypo- or hyperactivation within a specific hemisphere. In addition, when appropriate, we tested the independent effects of maternal depression and feeding groups on EEG asymmetry and EEG power scores. Finally, an exploratory linear regression analysis was conducted using imputed data to examine infant touch and the factors of interest (EEG asymmetry, temperament, maternal depression, infant touch at 1 month, and duration of breastfeeding) related to increases or decreases in maternal-directed infant affectionate touch behaviors.

\section{Results}

\section{Maternal Affectionate Touch}

A 1-way ANOVA revealed a significant effect by group in the proportion of maternal affectionate touch present at the 1 -month visit (see Table 2 for means, SDs, and $F$ and $p$ values). One-tailed $t$ tests indicated that M-BFND engaged in significantly more affectionate tactile behaviors than M-FFND $(t(69)=3.96, p<0.001 ; g=1.18)$ and M$\operatorname{FFD}(t(57)=2.35, p=0.01 ; g=0.96)$. The depressed groups also differed, with M-BFD showing more affectionate touch than M-FFD $(t(18)=2.16, p=0.03 ; g=0.93)$.

A similar ANOVA was conducted using the 3-month affectionate touch scores. Differences in maternal affectionate touch between groups at the 3-month visit were significant (Table 2). Post hoc comparisons suggested that the mothers in the nondepressed feeding groups and M-BFD provided more affectionate touch to their infants than M-FFD $(t(52)=2.27, p=0.015 ; g=0.92$ and $t(13)=$ $2.29, p=0.02 ; g=1.18$, respectively).

\section{Infant Affectionate Touch}

The 1-month infant affectionate touch composite variable was also analyzed using a 1-way ANOVA, which indicated no differences for the 4 groups overall ( $p>$ 0.05 ). Examination of the means (Table 2) showed that I-FFD performed the least touch.

Finally, a 1-way ANOVA on the infant's tactile behavior for each group revealed significant differences in AT-I for the 3-month visit. Post hoc comparisons revealed that I-BFND touched their mothers (Table 2) significantly more than I-FFND $(t(55)=2.71, p=0.004 ; g=0.83)$ and I-FFD at the 3-month visit $(t(48)=1.68, p=0.05 ; g=$ $0.68)$. There was no significant difference in infant affectionate touch between the nondepressed and depressed groups who breastfed.

\section{Touch and Temperament}

Correlational analyses showed a significant negative correlation between maternal-rated infant activity level at 
Table 3. Multivariate analyses: effects across age for asymmetry and power scores

\begin{tabular}{|c|c|c|c|c|}
\hline Multivariate effects & df & $F$ value & $\begin{array}{l}\text { Significance, } \\
p \text { value }\end{array}$ & $\begin{array}{l}\text { Effect size, } \\
\text { partial } \eta^{2}\end{array}$ \\
\hline \multicolumn{5}{|l|}{ EEG asymmetry across age $(N=66)$} \\
\hline Group $\times$ region & 3.62 & 4.00 & 0.01 & 0.19 \\
\hline Group $\times$ region $\times$ age & 3.62 & 5.00 & 0.004 & 0.24 \\
\hline \multicolumn{5}{|l|}{ EEG asymmetry at 1 month $(N=92)$} \\
\hline Group $\times$ region & 3.88 & 3.39 & 0.02 & 0.12 \\
\hline \multicolumn{5}{|l|}{ EEG asymmetry at 3 months $(N=73)$} \\
\hline Group $\times$ region & 3.69 & 4.14 & 0.009 & 0.18 \\
\hline \multicolumn{5}{|l|}{ EEG power across age $(N=66)$} \\
\hline Group $\times$ hemisphere & 3.62 & 2.60 & 0.05 & 0.12 \\
\hline Group $\times$ region $\times$ hemisphere & 3.62 & 4.00 & 0.01 & 0.19 \\
\hline Group $\times$ region $\times$ hemisphere $\times$ age & 3.62 & 5.00 & 0.004 & 0.24 \\
\hline \multicolumn{5}{|l|}{ EEG power at 1 month $(N=92)$} \\
\hline Group $\times$ region $\times$ hemisphere & 3.88 & 3.39 & 0.02 & 0.12 \\
\hline \multicolumn{5}{|l|}{ EEG power at 3 months $(N=73)$} \\
\hline Region $\times$ hemisphere & 3.67 & 4.35 & 0.007 & 0.19 \\
\hline Group $\times$ region $\times$ hemisphere & 3.69 & 4.14 & 0.009 & 0.18 \\
\hline
\end{tabular}

1 month and AT-I $(r(63)=-0.28, p=0.03)$; however, no other relationships emerged between mother or infant affectionate touch behaviors and ratings of temperament. A repeated measures Group (4 groups) by Age (2) MANOVA was conducted using infant temperament scores as the dependent variable. This analysis indicated a significant main effect for age (suggesting higher ratings at 3 months than at 1 month, $F(1,65)=60.73, p<0.000)$ and also showed that mothers rated their infants as higher in positive qualities than negative ones $(F(1,65)=$ $79.60, p<0.000)$. However, there were no significant between-group differences for maternal-reported temperament measures.

Although no predictions were made about correlations between maternal depression scores and temperament, we did find that higher depression scores were associated with higher activity levels and distress composites ( $r$ 's ranged from 0.25 to $0.31, p$ 's $<0.04$ ), across age. Further, longer breastfeeding duration was related to more mother and infant affectionate touch ( $r$ 's ranged from 0.23 to $0.42, p$ values $<0.03$ ), across age.

\section{EEG Analyses across Age}

One- and 3-month EEG Asymmetry Analysis

A series of repeated measures MANOVAs were conducted on EEG asymmetry scores with Group by Region by Age as between- and within-subjects factors. These analyses yielded several significant interaction effects (Table 3). In order to unpack these findings, analyses were conducted, separately for each region to assess changes across age.

ANOVAs between 1- and 3-month frontal EEG asymmetry indicated that 3-month and not 1-month groups $(p>0.05)$ differed. Examination of the means indicated that the depressed and breastfeeding group was similar to the nondepressed groups in showing left frontal EEG asymmetry, while the depressed and bottle-feeding group had right frontal EEG asymmetry (Table 4). Analyses with the central, the parietal, and the occipital regions were nonsignificant ( $p s>0.05$ ) across both ages. Further analyses were conducted to examine differences within age and then, if appropriate, to examine the contribution of feeding and maternal mood groups independently.

\section{Within Age 1-Month EEG Asymmetry}

The 1-month infant EEG asymmetry analysis yielded a significant Group $\times$ Region interaction effect $(F(3,88)$ $\left.3.39, p=0.02, \eta^{2}=0.11\right)$. Examining each region separately showed no statistically significant effects at 1 month. However, comparisons between depression (2) and feeding (2) groups on regional asymmetries at 1 month showed a main effect for depression group $(F(1$, $\left.86)=4.16, p=0.04, \eta^{2}=0.05\right)$ but not an effect for feeding group $(p<0.05)$, with the depressed group showing 
Table 4. EEG asymmetry across age in infants at 1 and 3 months of age

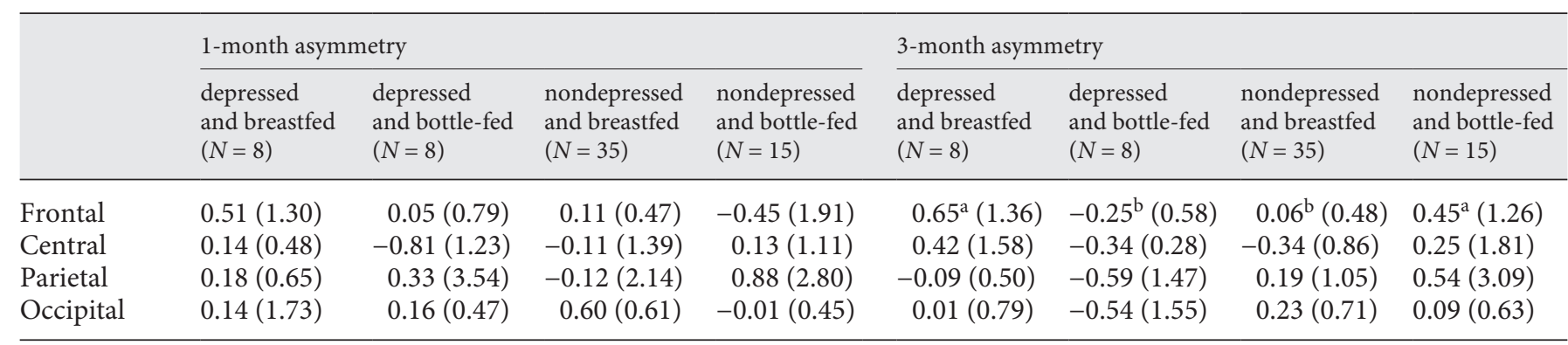

Values reflect mean EEG asymmetry data in the 3- to 6-Hz frequency band at 1 month and in the 6- to 9-Hz frequency band at 3 months. Standard deviations are in parentheses. Different letter superscripts in each row are significantly $(p<0.05)$ different from each other. Central, parietal and occipital region analyses at each age were non-significant.

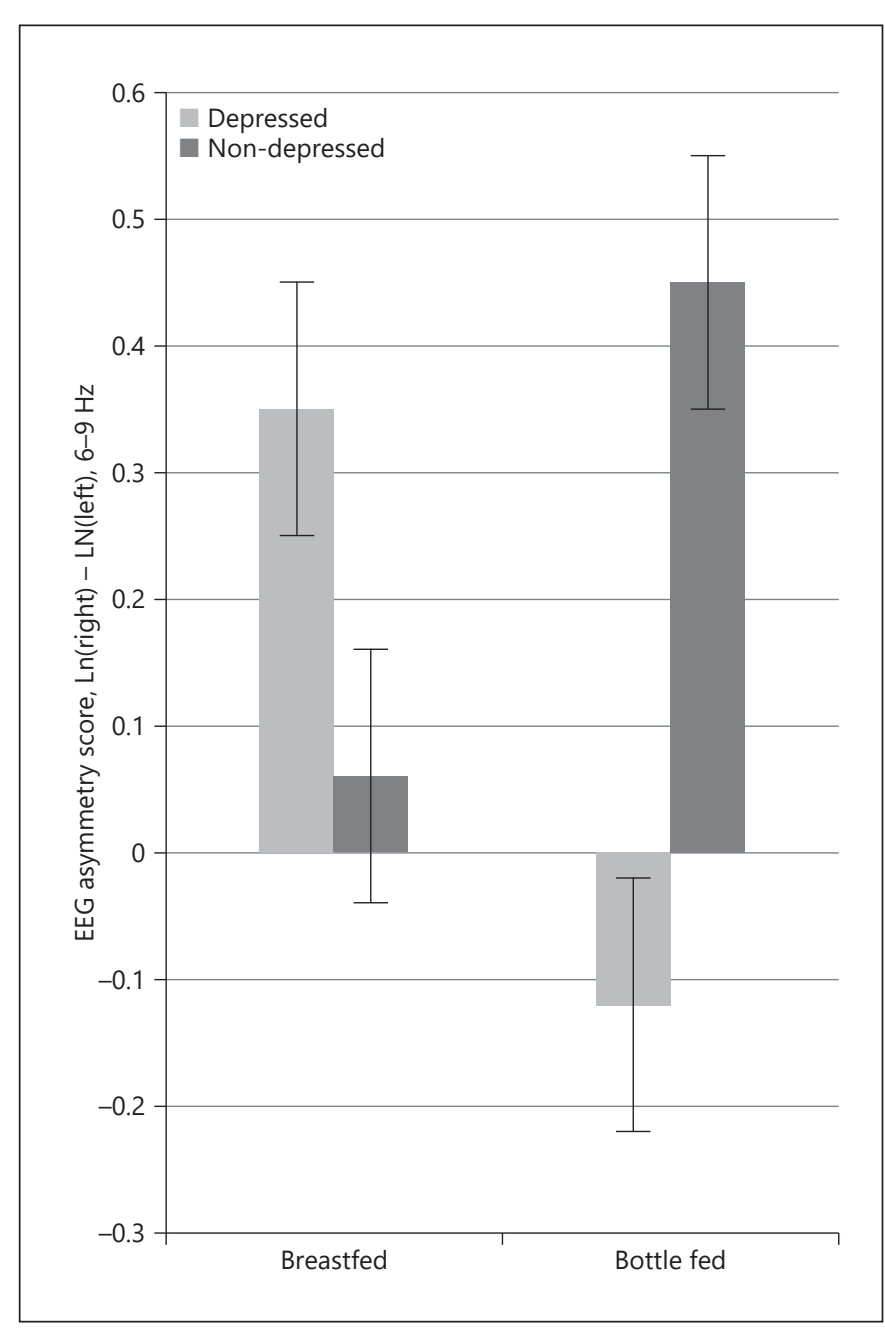

Fig. 1. Three-month frontal EEG asymmetry. The depressed, bottle-fed infant group is the only group at 3 months to display relative right hemispheric activation. overall greater relative right hemisphere EEG asymmetry $(\mathrm{M}=-0.56, \mathrm{SD}=0.32$ for the depressed group and $\mathrm{M}=$ $0.31(\mathrm{SD}=0.17)$ for the nondepressed group).

Within Age 3-Month EEG Asymmetry

For the 3-month infant's EEG asymmetry data, Group $\times$ Region analyses yielded a significant effect for Region $\left(F(3,67)=4.35, p=0.007, \eta^{2}=0.16\right)$ and a Group $\times$ Region interaction $\left(F(3,69)=4.14, p=0.009, \eta^{2}=0.15\right)$. An ANOVA comparing the 4 groups yielded a significant effect for only the frontal region $(F(3,69)=2.76, p=0.05$, $\left.\eta^{2}=0.11\right)$ but no effects for the other regions tested ( $p$ 's $>$ $0.05)$.

To further probe the differences for each group, an analysis comparing the depression (2) and feeding (2) groups separately showed main effects for feeding group $\left(F(1,60)=4.12, p=0.05, \eta^{2}=0.06\right)$ as well as depression and feeding group interactive effects $(F(1,60)=4.58, p=$ $\left.0.04, \eta^{2}=0.07\right)$ for the frontal region but not for other regions. Examination of means showed that the breastfeeding group had greater left frontal asymmetry $(M=0.32$; $\mathrm{SD}=0.27)$ and bottle feeders had greater right frontal asymmetry $(\mathrm{M}=-0.12 ; \mathrm{SD}=0.21)$. The interactive effects indicated that infants of depressed mothers who breastfed had left frontal asymmetry and those that bottle-fed had right frontal asymmetry. Yet comparisons between nondepressed groups show no significant differences $(p>0.05)$, as their frontal asymmetry scores were similar to those in the depressed, breastfeeding group (Fig. 1).

Across Age 1- and 3-Month EEG Power Analysis

In order to examine the contributions of each hemisphere, a Group $\times$ Region $\times$ Hemisphere $\times$ Age MANO- 
Table 5. EEG power across age in infants at 1 and 3 months of age

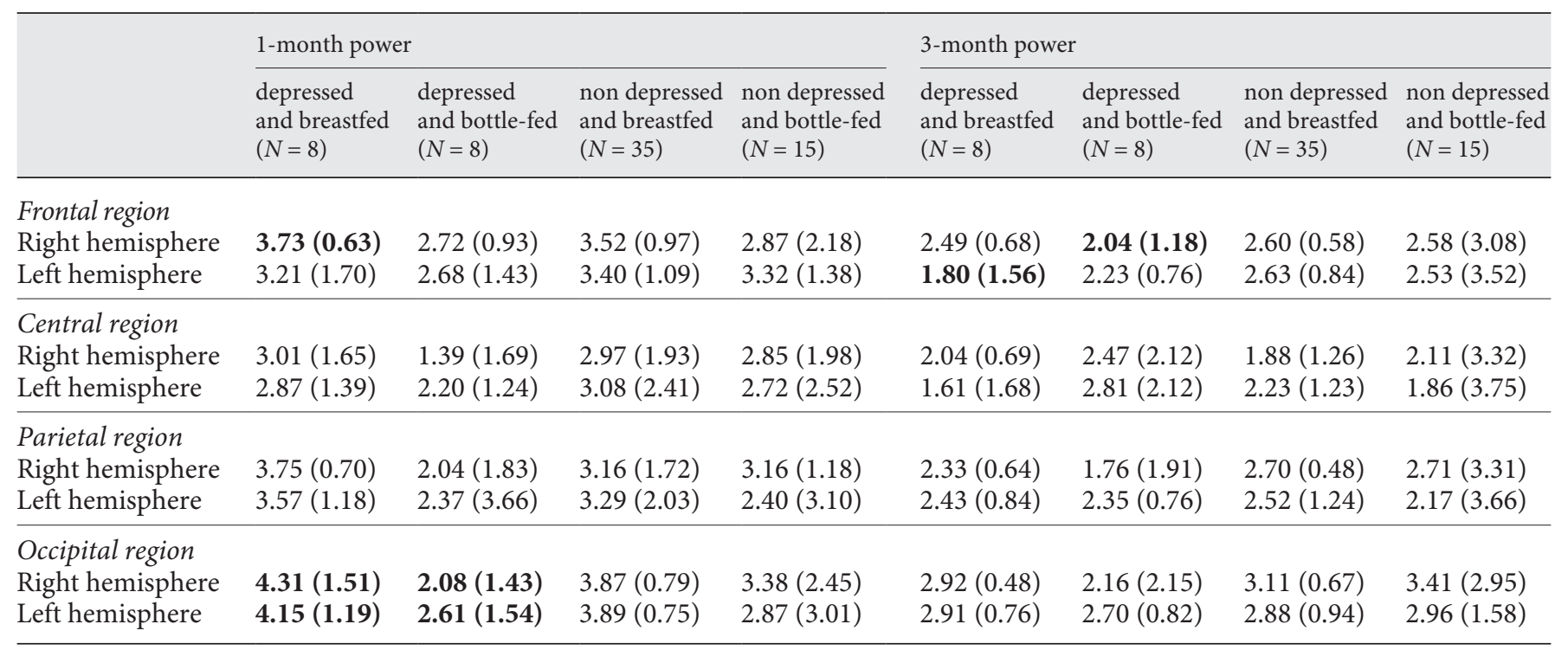

Values reflect mean EEG asymmetry data in the 3- to 6-Hz frequency band at 1 month and in the 6- to 9-Hz frequency band at 3 months. Standard deviations are in parentheses. Different letter superscripts in each row are significantly $(p<0.05)$ different from each other. Central, parietal and occipital region analyses at each age were non-significant.

VA was conducted on the natural log-transformed power scores. A number of main effects (Age, Region) and interactions emerged (Group $\times$ Hemisphere, Group $\times$ Region $\times$ Hemisphere, Group $\times$ Region $\times$ Age) including an overall 4-way interaction effect (Table 3 ). Due to the significant Age and Region main effects and to understand the 4-way interaction, separate analyses were conducted for each region and at each age.

Group by Hemisphere by Age MANOVAs for each region showed an Age effect for the frontal region (Table 5) but no other effects. Central and parietal region analyses were all nonsignificant. Of interest, however, analyses for the occipital region yielded a significant Group $\times$ Hemisphere interaction (Table 5), with mean values indicating less right hemisphere power in the depressed, bottle-feeding group and the most right hemisphere power in the depressed, breastfeeding group with a similar pattern for the left hemisphere.

Of significant interest, analyses utilizing power scores at both 1 and 3 months, comparing feeding and depression groups separately for the frontal region again, showed a main effect for age $\left(F(1,67)=12.54, p=0.001, \eta^{2}=0.16\right)$ and a depression group $\times$ feeding group $\times$ age $\times$ hemisphere interaction $\left(F(1,67)=5.71, p=0.02, \eta^{2}=0.08\right)$. As shown in Figure 2 (Table 5, bold values), the age changes in left hemisphere scores were most significant for the depressed breastfeeding group, with this group increasing in left hemisphere activity (lower power and hyperactivation of the left hemisphere) from 1 to 3 months. Further, the depressed bottle-feeding group showed changes but principally within the right hemisphere, with an increase in right frontal activity (lower power) across age.

\section{Within Age 1-Month EEG Power}

Separate Group $\times$ Region $\times$ Hemisphere analyses showed a main effect for Region $(F(3,86)=4.10, p=$ $\left.0.009, \eta^{2}=0.14\right)$ and a 3 -way Group $\times$ Region $\times$ Hemisphere interaction at 1 month $(F(3,88)=3.89, p=0.02$, $\left.\eta^{2}=0.11\right)$. No significant findings were uncovered for 1-month hemisphere and group activity in any of the separate regions.

\section{Within Age 3-Month EEG Power}

Separate Group $\times$ Region $\times$ Hemisphere analyses for the 3-month data showed a Region $\times$ Hemisphere interaction effect as well as a 3-way Group $\times$ Region $\times$ Hemisphere interaction at 3 months $(F(3,62)=3.89, p=0.02$, $\left.\eta^{2}=0.11 ; F(3,64)=4.14, p=0.009, \eta^{2}=0.18\right)$. Separate analyses were then conducted for each region at 3 months.

For the frontal region, a Group $\times$ Hemisphere ANOVA was significant for the 4 groups $(F(3,67)=3.34, p=$ $0.02, \eta^{2}=0.15$ ). Analyses showed significant group differ- 


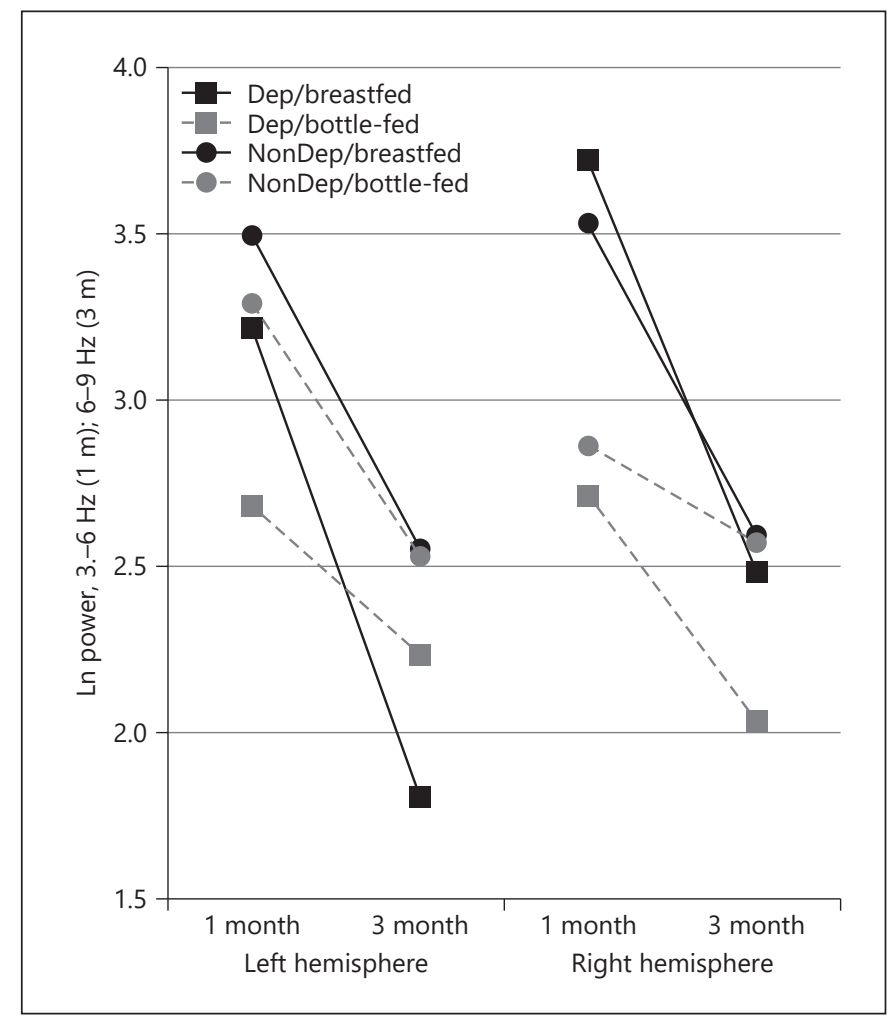

Fig. 2. Frontal region EEG power illustrating change in infant hemispheric activation across 1 and 3 months of age and between the 4 feeding/depression groups.

ences both in both right and left hemisphere EEG power scores for the 4 groups. Interestingly, I-BFD showed greater activation of the left hemisphere (lower power), whereas I-FFD showed more right hemisphere hyperactivation (Fig. 3). Infants in the nondepressed groups showed little differences in right and left power (left hemisphere: $\mathrm{M}=2.45$ [ $\mathrm{SD}=0.93$ ] and right hemisphere: $\mathrm{M}=2.42$ [SD = 0.92] for I-BFND) or, as expected, slightly more left hemisphere activity than right hemisphere power (left hemisphere: $\mathrm{M}=1.91$ [SD = 3.31] and right hemisphere: $\mathrm{M}=2.52$ [SD $=2.82]$ for I-FFND). No additional effects at 3 months of age were uncovered in the central, parietal, and occipital regions.

\section{Exploratory Analysis: Predicting Infant Touch}

Behavior

We chose to examine infant touch behaviors in a regression model using imputed data because multiple imputation is generally viewed as an appropriate statistical procedure for handling incomplete data sets [64]. The iterations were set to 5 . We used maternal depression sta-

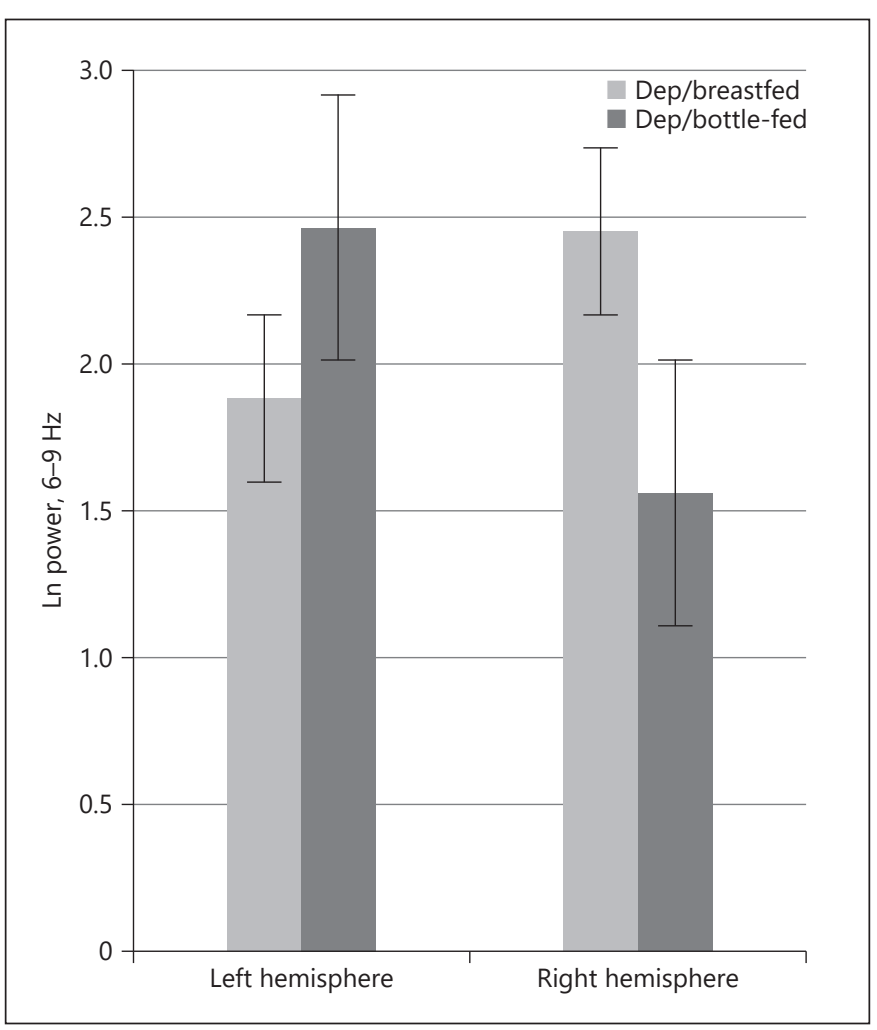

Fig. 3. Right and left hemispheric frontal EEG power across the 6-9 $\mathrm{Hz}$ bands for the depressed group at 3 months.

tus, days breastfeeding across the 3 months, infant frontal asymmetry at 3 months, positive temperament scales (i.e., the soothing and the smiling and laughing composites), and infant touch patterns at 1 and 3 months as variables to impute the missing data.

A multiple regression analysis using the imputed data was conducted to determine the predictability of the average time infants spent affectionately touching their mothers at 3 months using frontal EEG asymmetry scores, depression status, days breastfeeding, and the amount of time the infant spend touching the mother at 1 month, as the predictors. The overall regression analysis was significant $\left(F(6,106)=6.10-8.48\right.$, ps $<0.001, R^{2}=0.26-$ 0.32 ). Of the individual predictors, days breastfeeding (unstandardized $\beta=0.53, p=0.001$ ) and infant's average temperamental positive affect (unstandardized $\beta=11.74$, $p=0.004$ ) were significant predictors of the time infants spent affectionately touching their mothers at 3 months. The findings suggest that breastfeeding and temperamentally positive affect uniquely predict the development of infant affectionate touch patterns. 


\section{Discussion}

As experience with maternal mood and feeding pervade the infant's early environment, we chose to examine how these factors interact to affect mother-infant affectionate touch, focusing fastidiously on the key roles of temperament and EEG activation patterns. Overall, the findings indicate that (1) feeding method differentially affected outcomes for infants of depressed mothers (compared to nondepressed groups) in the quality of affectionate tactile patterns; (2) feeding method also affected EEG asymmetry and power in infants of depressed and nondepressed mothers, and (3) finally, an overall model was supported in which the infant's 3-month affectionate touch behavior was predicted by breastfeeding, temperament, frontal EEG asymmetry, maternal depression, and prior affectionate touch patterns. In particular, affectionate touch of mothers and infants varied by depression interacting with feeding type, with breastfeeding having a positive effect on both maternal and infant affectionate touch. Notably, EEG patterns of infants of depressed mothers showed right frontal asymmetry; however, shifts to greater left frontal activation (left frontal hyperactivation change) were found in those infants with stable breastfeeding experiences. The I-FFD group, alternatively, showed hyperactivation of the right hemisphere (as well as modest hypoactivation of the left hemisphere) at 3 months of age. In addition, our exploratory analysis revealed that infant breastfeeding duration and positive temperamental characteristics predicted infant affectionate touch patterns, suggesting that early infant experiences, and more broadly, their underlying neurochemical regulatory processes during feeding could influence the development of infant physiology and behavior, even for infants of depressed mothers.

\section{Quality of Touch: Maternal Affectionate Tactile Patterns}

Touch is an important component of social interactions, as it is a mechanism for conveying affective information within the mother-infant relationship [65]. Our findings demonstrated that feeding context was an important factor of affectionate touch behaviors for depressed mothers. At 1 month postpartum, depressed and breastfeeding mothers engaged in the most affectionate touch, whereas both bottle-feeding groups showed much lower levels of affectionate touch. Later at 3 months, MBFND engaged in the highest amount of affectionate touch. Most notably, across age, affectionate touch by the mothers in the depressed and breastfeeding group di- verged significantly from the touch patterns of M-FFD. The former group was more concordant with the nondepressed group. This finding, in particular, is of interest, as previous research has demonstrated that the styles of touch in depressed mothers are dysregulated [66, 67]. Breastfeeding appears to enhance the dyad's touch interactions even in depressed mother-infant pairs [3].

While there is controversy over whether or not breastfeeding has any long-term benefits on the mother-infant relationship outside of nutritional benefits for the infant [68], our findings revealed an association between breastfeeding and affectionate touch patterns in depressed mothers. One interpretation of our findings is that touch experiences during feeding are an especially important part of the developing patterns of behavior related to the emerging mother-infant relationship because of the amount of the time mothers and infants spend in the feeding context. Moreover, the quality of emotional care the mothers provide during feeding is important [69]. Thus, the current study provides preliminary evidence that the breastfeeding environment is associated with affectionate tactile patterns in both depressed and nondepressed dyads. This is in line with previous research linking breastfeeding to enhanced maternal responsiveness to infants' needs [70], and thus, the breastfeeding environment may ameliorate some of the negative associations found in the dyad's relationship for those with depressive symptoms.

\section{Quality of Touch: Infant Affectionate Tactile Patterns}

Social touch patterns are influenced bidirectionally, and therefore, it is important to consider the emerging patterns of infant touch $[3,71]$ as well as how the infant is influencing the parent's behaviors. By 3 months of age, I-BFD had developed touch patterns that were more in alignment with the nondepressed dyads. Conversely, IFFD displayed the lowest amounts of affectionate touch across age.

Notably, the pattern of results for several of our analyses revealed that increases in the number of days spent breastfeeding and more positive temperament characteristics, even for mothers with depressive symptoms, predicted AT-I behavior at 3 months. Typically, infants of depressed mothers engage in more reactive types of touch or use of self-touch [72], likely because they have been found to be less soothed by maternal touch $[49,50]$. In the present study, reduced infant touch toward their mothers was observed only when the infants were in the depressed and bottle-fed group. Our research offers a unique contribution to the literature base on mother-infant dyads 
because it provides evidence that a stable breastfeeding environment may attenuate the behavioral risk associated in past studies with maternal depression and redirect the trajectory of socio-emotional development of their atrisk infants across the early months of life [73].

Breastfeeding is an integral part of the early motherinfant relationship that may influence both the mother's and the infant's behavioral outcomes as well as their temperamental characteristics and neurophysiology. Although our study was not designed to specifically test any model of the development of temperament, our neurophysiological and behavioral findings are, in part, associated with the FET model of temperament [24]. Under the FET model, dyadic touch, maternal depressive symptoms, breastfeeding, and their underlying neurobiological systems can be viewed as a dynamical system that emerges across infancy into behavioral and physiological dimensions associated with infant temperament. The FET model provides a comprehensive framework for developmental research incorporating temperament, behavior, and neurophysiology. Thus, the FET model should be used as a framework in future breastfeeding research examining the environmental context and its relation to multiple domains (physiological, behavioral, and hormonal) related to infant temperament.

\section{EEG Asymmetry and Power in Infants}

In addition to the tactile behavior changes, the infants in this study displayed differential brain activation patterns (EEG asymmetry and power) as a function of maternal depression and feeding group status. Consistent with previous studies $[3,5,6,8,34,74]$, infants of depressed mothers showed right frontal EEG asymmetry patterns at the 3-month visit. Indeed, a recent review of the literature from the past 30 years revealed that depression is associated with dysregulated frontal region activation [33]. Specifically, researchers consistently have found less left activation, more right activity, and/or right frontal asymmetry patterns evident in basal and stimulus-associated EEG patterns. This neurological substrate has also been recorded in infants who are offspring of depressed mothers and potentially at risk for depressive disorders [37], potentially transmitted from their parent's genetic material and/or from the early environmental influences $[75,76]$. An important caveat, however, is that the infant's frontal region development is protracted, and it is unclear whether measures of EEG asymmetry and power can be reliable indices of infant brain functioning in depressed groups when the infants themselves are not depressed, rather their early environmental experiences are inundated with context-linked risk. Nonetheless, meta-analyses [37] have identified early brain activation patterns in infants of depressed mothers as a predictive physiological marker for the risk for mood disorders later in life. Examining the link between these factors is critical for understanding brain maturation as well as the experiential factors that can impact changes across development. The novelty in the findings presented here is that not only were the infants' EEG patterns affected by their mother's depression status but also stable breastfeeding experience interacted with the depression group to impact EEG patterns across early development. Thus, while our data are not causal, interacting portions of the infant's social context as well as the infant's own effect on their environment could be associated with the infant's behavioral, temperamental, and physiological development and functioning.

Importantly, our data also verify the necessity of examining detailed alterations in EEG power scores across development [77], as we documented a shift in hemispheric activity across age in the frontal region as well as revealing meaningful group differences in the trajectory of neuroplasticity across development. I-BFD showed the largest shift to more activation in the left frontal region, whereas I-FFD showed change indicative of left hemisphere hypoactivation and right hemisphere hyperactivation. It should be noted that this latter group was also the only group that experienced less affectionate touch and displayed less touch toward their mothers. Increases in right frontal alpha activity (lower alpha power) have been linked theoretically and empirically in typical and at-risk groups to younger periods in development, increases in negative affect, and dysregulation in emotional development [78-80]. In addition, hyperactivation of the right hemisphere of the frontal lobe has been coupled with more withdrawal-type behavioral tendencies and motivations, thereby encompassing more primitive emotions associated with the flight response of behavior, such as fear, sadness, and disgust $[81,82]$.

While not all studies have found statistically significant patterns of stable frontal EEG patterns before 3 months in infants of depressed mothers [36], it seems clear that experience with depressed mothers throughout infancy is associated with higher risk of right frontal asymmetry, potentially due to heightened right and lower left frontal activation patterns. In the current study, left frontal asymmetry in infants was associated with having a nondepressed mother and infant care experiences in the form of stable breastfeeding. Notably, left frontal activity has been associated with advancing maturation, approach 
motivations, positive emotions, as well as higher order processing skills [29, 83-88].

Our findings are in line with previous breastfeeding research. For example, researchers have found that 1 -week-old breastfed infants demonstrate superior neurobehavioral functioning [89] relative to formula-fed infants. Studies examining structural and functional neurodevelopment have shown that breastfeeding favorably influences a plethora of brain processes including more advanced cognitive skills [90-93], more optimal evoked response potentials within visual and auditory areas [94], increased leptin [95], smaller ventricular volumes, larger head circumferences [96], and brain maturation earlier in development through the impact on myelination and white matter development [97]. While Jing and colleagues [98] showed that breastfeeding influences EEG power shifts toward more mature frequency patterns earlier in development, no study before ours has examined the changes in left/right power and the shift in power that we uncovered in breastfed infants who also experienced the risk of maternal depression.

Two points should be considered here (1) that these were younger infants who are expected to have a predominantly right frontal EEG pattern and (2) that as the infants developed, the changes and interaction with left frontal region was affected by both positive (affectionate touch and positive temperament) and negative (maternal depression) infant experiences. This potentially corroborates the idea that experience-dependent plasticity is at work earlier than previous theories have claimed [80,99], in that, the infant's left frontal region interacted with the right as a function of experiences, likely through both neurohormonal and behavioral pathways. Thus, significant and stable experiences in caretaking, like those seen within breastfeeding may produce advantages in brain maturation for infants of depressed mothers. The present study demonstrates that the right frontal asymmetry in EEG patterns remains dominant in infants whose mothers are depressed but only for those who are bottle-fed as early as 3 months of age. The plasticity of the infant brain is, we expect, vulnerable to environmental interactions and appears to be negatively affected by the presence of maternal depression [100] and the lack of stable breastfeeding patterns and more optimally affected in I-BFD toward more left frontal EEG, like their nondepressed peers.

The EEG patterns noted in central, parietal, and occipital regions of the brain were less robust and not consistent across the study. However, there was one notable effect within the occipital region, specifically that the I-

Touch and Neurodevelopment
FFD showed more right hemisphere activation than IBFD; however, there were no significant findings for the left hemisphere. These findings, in the occipital region, could also suggest that there are developing sensorylinked EEG activation alterations as a result of breastfeeding [101]. Yet, the findings in other regions overall were less consistent than those uncovered within the frontal region and thus need replication for their meaning to become clear.

\section{Future Directions}

Our study did not measure other neurodevelopmental patterns associated with infant brain development and socio-emotional interactions between dyads. One possibility for the findings presented here is that breastfeeding and affectionate touch patterns influence neurohormonal responses associated with breastfeeding. Incorporating behavioral, physical affective, neurophysiological, and neurohormonal variables in future research will further elucidate the underlying mechanisms guiding the development of the mother-infant relationship and individual functioning. Furthermore, incorporating more neurohormonal measures in future research will allow a more comprehensive measure of infant temperament and the mother-infant relationship that is not reliant on maternal-reported infant temperament while also more fully addressing models of temperament including the FET model.

For example, because of oxytocin's role in the affectionate relationship between the mother-infant pair, researchers have theorized that healthy mother-infant touch may signal more sensitive caretaking, may serve to form and strengthen secure attachment relationships, and has been related to quicker stress-cortisol recovery in infants $[102,103]$. In addition to the link between oxytocin, researchers have also implicated the mu-opioid receptor (MOR) system in the neurobiology of affiliative touch (for a review see [104]). The link between MOR, but not oxytocin, activity and touch may, in part, be mediated genetically [105]. Future research on mother-infant touch, the involvement of the MOR system, and variations of the MOR and OXT receptor genes may be of particular interest to researchers, as the context of the mother-infant relationship represents a unique behavioral environment.

Other neurobiological processes underlying depression should also be studied in the context of dyadic feeding and touch. Noradrenergic activity, for example, influences depressive symptoms [106] but is downregulated in response to oxytocin release [107]. For depressed moth- 
ers, the hormonal releases of oxytocin and vasopressin (and thus the downregulation of the HPA axis) associated with breastfeeding may not only increase maternal sensitivity and affectionate infant touch early in life but also indirectly promote more mature brain processing, a secure attachment relationship, and bolster an individual's resilience in stress regulation across development [70, 103], thereby dynamically influencing infant temperament. These are all important benefits, especially for the infants of depressed mothers who are at risk for dysregulated developmental patterns both physiologically and psychologically.

\section{Limitations}

It is also important to note the three limitations of measuring frontal activation using EEG in young infants. First, and as mentioned previously, the frontal region is not fully developed at 1 month of age; therefore, EEG recordings could reflect inexact frequency band values (3-6 $\mathrm{Hz}$ may not adequately represent alpha activity in 1-month-old infants) or possibly subcortical activity. Second, there are no studies that specify the most reliable and valid EEG reference site in infants. Third, researchers have interpreted brain activation patterns along the power spectra differently. Studies have shown that there is a shift in power across the frequency spectra from early infancy to childhood and adolescence [108], and this must be taken into account to understand brain development in the context of early experiences. For example, in previous studies, a broad band $(3-13 \mathrm{~Hz})$ was used to examine EEG activity in the frontal lobe in the studies of 1-week and 1-month-old infants $[5,8]$. Later work showed that the frequency spectra shifts from 3-9 Hz to $4-9 \mathrm{~Hz}$ across the first 6 months even in infants of depressed mothers [2]. Shifts in EEG power and asymmetry scores may also be indicative of experiences or normative patterns of brain development that are not concordant with individual variation in infant behavioral development nor associated with maternal risk factors. Therefore, EEG recordings must be interpreted with caution, as alternative explanations for EEG pattern shifts in infants exist.

The classification of the feeding groups and the limited numbers of participants in the depressed and breastfeeding group along with the attrition in depressed groups were limitations of our study. The classification of the feeding groups was conservative, as we were particularly interested in how breastfeeding experiences affect touch and brain development. Future studies may utilize a more dynamic classification of feeding status to include mothers who employ variation in their feeding method across infancy to determine the point at which there are significant outcomes in infant behaviors and physiology. Furthermore, the data were collected in a seminaturalistic environment (laboratory setting). Future studies need to incorporate measures of feeding and touch behaviors in the participants' homes. Additionally, we had fewer M-BFD than we did M-BFND. Recruiting and retaining depressed mothers and their infants is always difficult, and this was exacerbated by the need to find depressed mothers with stable breastfeeding patterns as depressed mothers are less likely to initiate and maintain breastfeeding [109]. In future research, it may be prudent to provide physicians, health-care professionals, mental health workers, and infant care specialists with additional training and support for breastfeeding dyads in order to encourage more stable breastfeeding even in depressed mothers.

\section{Conclusion}

The findings of the current study provide preliminary evidence that feeding method and maternal depressive symptoms appear to interact to impact the socio-emotional relationship between the mother and infant as well as the infant's temperamental and neurodevelopmental patterns. Early experience across early infancy was differentially associated with affectionate touch patterns and more mature EEG patterns (i.e., left frontal activation), even in infants of depressed and breastfeeding mothers. Infants of depressed and breastfeeding mothersshowed neither behavioral nor brain development dysregulation previously found in infants of depressed mothers $[8,37]$, with only the depressed and bottle-feeding group demonstrating right frontal EEG asymmetry and more right along with less left hemisphere activation across development. Ultimately, the current study provides evidence that the sensitive caretaking that occurs, even for mothers with postnatal depression in the context of more predominant breastfeeding, may redirect neurophysiological, temperamental, and socio-emotional risk through dyadic tactile experiences across early development.

\section{Acknowledgements}

We would like to thank the families who participated in this study. We would also like to thank all the students at the Florida Atlantic University at Jupiter who helped with data collection and coding. Funding for this project was from an NIMH grant (\#MH61888) to N.A.J. 


\section{Statement of Ethics}

The research was ethically conducted in accordance with the World Medical Association Declaration of Helsinki and the APA ethical guidelines.

\section{Conflict of Interest Statement}

The authors have no conflicts of interest to declare.

\section{Funding Sources}

This research was funded by an NIMH grant (\#MH61888) awarded to N.A.J.

\section{Author Contributions}

J.S.H.: manuscript, data collection, and data analysis. N.A.J.: research funding, manuscript, data collection, and data analysis. K.D.M.: manuscript editing and funding. M.P.: data collection and manuscript editing.

\section{References}

1 Beebe B, Lachmann F, Jaffe J, Markese S, Buck $\mathrm{KA}$, Chen $\mathrm{H}$, et al. Maternal postpartum depressive symptoms and 4-month mother-infant interaction. Psychoanal Psychol. 2012 29(4):383-407.

2 Diego MA, Jones NA, Field T. EEG in 1-week, 1-month and 3-month-old infants of depressed and non-depressed mothers. Biol Psychol. 2010;83(1):7-14.

3 Jones NA, McFall BA, Diego MA. Patterns of brain electrical activity in infants of depressed mothers who breastfeed and bottle feed: the mediating role of infant temperament. Biol Psychol. 2004;67(1-2):103-24.

4 Jones N, Field T, Fox NA, Davalos M, Lundy B, Hart S. Newborns of mothers with depressive symptoms are physiologically less developed. Infant Behav Dev. 1998;21(3):537-41.

5 Jones NA, Field T, Fox NA, Lundy B, Davalos M. EEG activation in 1-month-old infants of depressed mothers. Dev Psychopathol. 1997; 9(3):491-505.

6 Field T, Fox NA, Pickens J, Nawrocki T. Relative right frontal EEG activation in 3- to 6-month-old infants of "depressed" mothers. Dev Psychol. 1995;31(3):358-63.

7 Ko JY, Rockhill KM, Tong VT, Morrow B, Farr SL. Trends in Postpartum Depressive Symptoms: 27 States, 2004, 2008, and 2012. MMWR Morb Mortal Wkly Rep. 2017;66(6): 153-8.

8 Jones NA, Field T, Fox NA, Davalos M, Malphurs J, Carraway K, et al. Infants of intrusive and withdrawn mothers. Infant Behav Dev. 1997;20(2):175-86.

9 Lundy B, Field T, Pickens J. Newborns of mothers with depressive symptoms are less expressive. Infant Behav Dev. 1996;19(4): 419-24.

10 McGrath JM, Records K, Rice M. Maternal depression and infant temperament characteristics. Infant Behav Dev. 2008;31(1):71-80.

11 Granat A, Gadassi R, Gilboa-Schechtman E, Feldman R. Maternal depression and anxiety, social synchrony, and infant regulation of negative and positive emotions. Emotion. 2017;17(1):11-27.
12 Netsi E, Pearson RM, Murray L, Cooper P, Craske MG, Stein A. Association of persistent and severe postnatal depression with child outcomes. JAMA Psychiatry. 2018;75(3):247.

13 Gotlib IH, Joormann J, Foland-Ross LC. Understanding familial risk for depression: a $25-$ year perspective. Perspect Psychol Sci. 2014; 9(1):94-108.

14 Huot RL, Brennan PA, Stowe ZN, Plotsky PM, Walker EF. Negative affect in offspring of depressed mothers is predicted by infant cortisol levels at 6 months and maternal depression during pregnancy, but not postpartum. In: Yehuda R, McEwen B, editors. Annals of the New York academy of sciences: vol. 1032. Biobehavioral stress response: protective and damaging effects. New York: New York Academy of Sciences; 2004. p. 234-6.

15 Greenough WT, Black JE, Wallace CS. Experience and brain development. Child Dev. 1987;58(3):539.

16 Siegel DJ. The developing mind. New York: The Guilford Press; 1999.

17 Calkins SD, Fox NA, Marshall TR. Behavioral and physiological antecedents of inhibited and uninhibited behavior. Child Dev. 1996; 67(2):523-40

18 Bernier A, Calkins SD, Bell MA. Longitudinal associations between the quality of motherinfant interactions and brain development across infancy. Child Dev. 2016;87(4):115974.

19 Jones N, Field T, Davalos M. Massage therapy attenuates right frontal EEG asymmetry in one-month-old infants of depressed mothers. Infant Behav Dev. 1998;21(3):527-30.

20 Fox NA, Calkins SD, Bell MA. Neural plasticity and development in the first two years of life: evidence from cognitive and socioemotional domains of research. Dev Psychopathol. 1994;6(4):677-96.

21 Rothbart MK. Measurement of temperament in infancy. Child Dev. 1981;52(2):569-78.

22 Saby JN, Marshall PJ. The utility of EEG band power analysis in the study of infancy and early childhood. Dev Neuropsychol. 2012;37(3): 253-73.
23 Sulis W. Assessing the continuum between temperament and affective illness: psychiatric and mathematical perspectives. Philos Trans R Soc Lond B Biol Sci. 2018;373(1744):1-9.

24 Trofimova I, Robbins TW. Temperament and arousal systems: a new synthesis of differential psychology and functional neurochemistry. Neurosci Biobehav Rev. 2016 May;64:382-402.

25 World Health Organization [Internet]. Infant and young child feeding: model chapter for textbooks for medical students and allied health professionals; session two: the physiological basis of breastfeeding. 2009. Geneva. Available from: https://www.ncbi.nlm.nih. gov/books/NBK148970/

26 Petersson M, Uvnäs-Moberg K, Erhardt S, Engberg G. Oxytocin increases locus coeruleus alpha 2-adrenoreceptor responsiveness in rats. Neurosci Lett. 1998;255(2):115-8.

27 Calkins SD, Fox NA, Marshall TR. Behavioral and physiological antecedents of inhibited and uninhibited behavior. Child Dev. 1996; 67(2):523-40.

28 Fox NA, Schmidt LA, Henderson HA, Marshall PJ. Developmental psychophysiology: conceptual and methodological issues. 3rd ed. In: Cacioppo JT, LG Tassinary LG, Berntson GG, editors. Handbook of psychophysiology. New York: Cambridge University Press; 2007. p. 453-81.

29 Zhu C, Guo X, Jin Z, Sun J, Qiu Y, Zhu Y, et al. Influences of brain development and ageing on cortical interactive networks. Clin Neurophysiol. 2011;122(2):278-83.

30 Bell MA, Fox NA. Cognition and affective style: individual differences in brain electrical activity during spatial and verbal tasks. Brain Cogn. 2003;53(3):441-51.

31 Conrad NJ, Schmidt LA, Niccols A, Polak CP, Riniolo TC, Burack JA. Frontal electroencephalogram asymmetry during affective processing in children with Down syndrome: a pilot study. J Intellect Disabil Res. 2007; 51(Pt 12):988-95.

32 Gustafsson HC, Grieve PG, Werner EA, Desai P, Monk C. Newborn electroencephalographic correlates of maternal prenatal depressive symptoms. J Dev Orig Health Dis. 2018 Aug; 9(4):381-5. 
33 Bruder GE, Stewart JW, McGrath PJ. Right brain, left brain in depressive disorders: clinical and theoretical implications of behavioral, electrophysiological and neuroimaging findings. Neurosci Biobehav Rev. 2017;78:17891.

34 Field T, Diego M. Maternal depression effects on infant frontal EEG asymmetry. Int J Neurosci. 2008;118(8):1081-108.

35 Marshall PJ, Fox NA. Infant EEG and ERP in relation to social and emotional development. In: de Haan M, editor. Infant EEG and eventrelated potentials. New York: Psychology Press; 2007. p. 227-50.

36 Lusby CM, Goodman SH, Bell MA, Newport DJ. Electroencephalogram patterns in infants of depressed mothers. Dev Psychobiol. 2014; 56(3):459-73.

37 Peltola MJ, Bakermans-Kranenburg MJ, Alink LR, Huffmeijer R, Biro S, van IJzendoorn $\mathrm{MH}$. Resting frontal EEG asymmetry in children: meta-analyses of the effects of psychosocial risk factors and associations with internalizing and externalizing behavior. Dev Psychobiol. 2014;56(6):1377-89.

38 Diego MA, Field T, Hernandez-Reif M, Cullen C, Schanberg S, Kuhn C. Prepartum, postpartum, and chronic depression effects on newborns. Psychiatry. 2004;67(1):63-80.

39 Diego MA, Field T, Jones NA, HernandezReif M. Withdrawn and intrusive maternal interaction style and infant frontal EEG asymmetry shifts in infants of depressed and nondepressed mothers. Infant Behav Dev. 2006; 29(2):220-9.

40 Jones NA, Field T, Almeida A. Right frontal EEG asymmetry and behavioral inhibition in infants of depressed mothers. Infant Behav Dev. 2009;32(3):298-304.

41 Ferber SG, Feldman R, Makhoul IR. The development of maternal touch across the first year of life. Early Hum Dev. 2008;84(6):36370.

42 Feldman R, Eidelman AI. Direct and indirect effects of breast milk on the neurobehavioral and cognitive development of premature infants. Dev Psychobiol. 2003;43(2):109-19.

43 Gallace A, Spence C. The science of interpersonal touch: an overview. Neurosci Biobehav Rev. 2010;34(2):246-59.

44 Feldman R, Singer M, Zagoory O. Touch attenuates infants' physiological reactivity to stress. Dev Sci. 2010;13(2):271-8.

45 Hertenstein MJ, Campos JJ. Emotion regulation via maternal touch. Infancy. 2001;2(4) 549-66.

46 Blass EM, Ciaramitaro V, Barr RG. A new look at some old mechanisms in human newborns: taste and tactile determinants of state, affect, and action. Monogr Soc Res Child Dev. 1994;59(1):I-81.

47 Brauer J, Xiao Y, Poulain T, Friederici AD, Schirmer A. Frequency of maternal touch predicts resting activity and connectivity of the developing social brain. Cereb Cortex. 2016;26(8):3544-52.
48 Tuulari JJ, Scheinin NM, Lehtola S, Merisaari H, Saunavaara J, Parkkola R, et al. Neural correlates of gentle skin stroking in early infancy. Dev Cogn Neurosci. 2019;35:36-41.

49 Field T, Hernandez-Reif M, Diego M, Feijo L, Vera Y, Gil K, et al. Still-face and separation effects on depressed mother-infant interactions. Infant Ment Health J. 2007;28(3):31423.

50 Boyd RC, Zayas LH, McKee MD. Mother-infant interaction, life events and prenatal and postpartum depressive symptoms among urban minority women in primary care. Matern Child Health J. 2006 Mar;10(2):139-48.

51 Jones NA, Gagnon C, Mize KD. Nurturing touch is beneficial for depressed mothers and their infants. Paper presented at: the International Conference of infant studies. Japan: Kyoto; 2006 Jun 20-22.

52 Malphurs JE, Raag T, Field T, Pickens J, Pelaez-Nogueras M. Touch by intrusive and withdrawn mothers with depressive symptoms. Early Dev Parenting. 1996;5(2):111-5.

53 Lavelli M, Poli M. Early mother-infant interaction during breast- and bottle-feeding. Infant Behav Dev. 1998;21(4):667-83.

54 Field T, Diego M, Hernandez-Reif $M$, Figueiredo B, Ezell S, Siblalingappa V. Depressed mothers and infants are more relaxed during breastfeeding versus bottle feeding interactions: brief report. Infant Behav Dev. 2010;33(2):241-4.

55 Weaver JM, Schofield TJ, Papp LM. Breastfeeding duration predicts greater maternal sensitivity over the next decade. Dev Psychol. 2018;54(2):220-7.

56 Field T, Hernandez-Reif M, Feijo L. Breastfeeding in depressed mother-infant dyads. Early Child Dev Care. 2002;172(6):539-45.

57 Field T. Postpartum depression effects on early interactions, parenting, and safety practices: a review. Infant Behav Dev. 2010;33(1): $1-6$.

58 Radloff LS. The CES-D scale. Appl Psychol Meas. 1977;1(3):385-401.

59 Noel-Weiss J, Boersma S, Kujawa-Myles S. Questioning current definitions for breastfeeding research. Int Breastfeed J. 2012;7(1):9.

60 Polan HJ, Ward MJ. Role of the mother's touch in failure to thrive: a preliminary investigation. J Am Acad Child Adolesc Psychiatry. 1994;33(8):1098-105.

61 Moszkowski RJ, Stack DM. Infant touching behaviour during mother-infant face-to-face interactions. Inf Child Develop. 2007;16(3): 307-19.

62 Cuevas K, Bell MA. EEG and ECG from 5 to 10 months of age: developmental changes in baseline activation and cognitive processing during a working memory task. Int J Psychophysiol. 2011;80(2):119-28.

63 Lindsley DB. A longitudinal study of the occipital alpha rhythm in normal children: frequency and amplitude standards. Pedagog Semin J Genet Psychol. 1939;55(1):197-213.
64 Young R, Johnson DR. Handling missing values in longitudinal panel data with multiple imputation. Fam Relat. 2015;77(1):277-94.

65 Waters SF, West TV, Karnilowicz HR, Mendes WB. Affect contagion between mothers and infants: examining valence and touch. J Exp Psychol Gen. 2017;146(7):1043-51.

66 Egmose I, Cordes K, Smith-Nielsen J, Væver MS, Køppe S. Mutual regulation between infant facial affect and maternal touch in depressed and nondepressed dyads. Infant Behav Dev. 2018;50:274-83.

67 Herrera E, Reissland N, Shepherd J. Maternal touch and maternal child-directed speech: effects of depressed mood in the postnatal period. J Affect Disord. 2004;81(1):29-39.

68 Jansen J, Weerth C, Riksenwalraven J. Breastfeeding and the mother-infant relationship: a review. Dev Rev. 2008;28(4):503-21.

69 Smith JP, Ellwood M. Australian Centre for Economic Research on Health (ACERH) [Internet]. Where does a mothers' day go? Preliminary estimates from the Australian Time Use Survey of New Mothers. 2006. Available from: http://regnet.anu.edu.au/sites/default/ files/uploads/2016-01/Smith\%202006\%20 Ellwood\%202006\%20Where \%20does\%20 a\%20mothers\%27\%20day\%20go\%20ACERH_RR1.pdf.

70 Britton JR, Britton HL, Gronwaldt V. Breastfeeding, sensitivity, and attachment. Pediatrics. 2006;118(5):e1436-43.

71 Feldman R. The neurobiology of human attachments. Trends Cogn Sci. 2017;21(2):8099.

72 Moszkowski RJ, Stack DM, Girouard N, Field TM, Hernandez-Reif M, Diego M. Touching behaviors of infants of depressed mothers during normal and perturbed interactions. Infant Behav Dev. 2009;32(2):183-94.

73 Dias CC, Figueiredo B. Breastfeeding and depression: a systematic review of the literature. J Affect Disord. 2015;171:142-54.

74 Dawson G, Frey K, Panagiotides H, Osterling J, Hessl D. Infants of depressed mothers exhibit atypical frontal brain activity: a replication and extension of previous findings. Child Psychol Psychiatry. 1997;38(2):179-86.

75 Anokhin AP, Heath AC, Myers E. Genetic and environmental influences on frontal EEG asymmetry: a twin study. Biol Psychol. 2006; 71(3):289-95.

76 Fox NA, Rutter M. Introduction to the special section on the effects of early experience on development. Child Dev. 2010;81(1):23-7.

77 Gartstein MA, Hancock GR, Potapova NV, Calkins SD, Bell MA. Modeling development of frontal electroencephalogram (EEG) asymmetry: sex differences and links with temperament. Dev Sci. 2020 Jan;23(1):e12891-17.

78 Grossmann T. Mapping prefrontal cortex functions in human infancy. Infancy. 2013; 18(3):303-24.

79 Hecht D. Depression and the hyperactive right-hemisphere. Neurosci Res. 2010;68(2): 77-87. 
80 Schore AN. Dysregulation of the right brain: a fundamental mechanism of traumatic attachment and the psychopathogenesis of posttraumatic stress disorder. Aust N Z J Psychiatry. 2002;36(1):9-30.

81 Buss KA, Schumacher JR, Dolski I, Kalin NH Goldsmith HH, Davidson RJ. Right frontal brain activity, cortisol, and withdrawal behavior in 6-month-old infants. Behav Neurosci. 2003;117(1):11-20.

82 Davidson RJ, Fox NA. Asymmetrical brain activity discriminates between positive and negative affective stimuli in human infants. Science. 1982;218(4578):1235-7.

83 Blanton RE, Levitt JG, Thompson PM, Narr KL, Capetillo-Cunliffe L, Nobel A, et al. Mapping cortical asymmetry and complexity patterns in normal children. Psychiatry Res. 2001 Jul;107(1):29-43.

84 Harmon-Jones E, Gable PA, Peterson CK. The role of asymmetric frontal cortical activity in emotion-related phenomena: a review and update. Biol Psychol. 2010 Jul;84(3):45162.

85 He BJ, Zempel JM, Snyder AZ, Raichle ME. The temporal structures and functional significance of scale-free brain activity. Neuron. 2010 May;66(3):353-69.

86 Lavelli M, Fogel A. Developmental changes in the relationship between the infant's attention and emotion during early face-to-face communication: the 2-month transition. Dey Psychol. 2005;41(1):265-80.

87 Licata M, Paulus M, Kühn-Popp N, Meinhardt J, Sodian B. Infant frontal asymmetry predicts child emotional availability. Int $\mathrm{J} \mathrm{Be}-$ hav Dev. 2015 Nov;39(6):492-6.

88 Poole BD, Gable PA. Affective motivational direction drives asymmetric frontal hemisphere activation. Exp Brain Res. 2014 Jul; 232(7):2121-30.

89 Hart S, Boylan LM, Carroll S, Musick YA Lampe RM. Brief report: breast-fed oneweek-olds demonstrate superior neurobehavioral organization. J Pediatr Psychol. 2003 Dec 1;28(8):529-34.
90 Eidelman AI. Breastfeeding mitigates a disaster. Breastfeed Med. 2013 Jun;8(3):344-5.

91 Oddy WH, Kendall GE, Blair E, de Klerk NH, Stanley FJ, Landau LI, et al. Breast feeding and cognitive development in childhood: a prospective birth cohort study. Paediatr Perinat Epidemiol. 2003 Jan;17(1):81-90.

92 Pivik RT, Andres A, Badger TM. Diet and gender influences on processing and discrimination of speech sounds in 3- and 6-monthold infants: a developmental ERP study. Dev Sci. 2011 Jul;14(4):700-12.

93 Sloan S, Stewart M, Dunne L. The effect of breastfeeding and stimulation in the home on cognitive development in one-year-old infants. Child Care Pract. 2010 Apr;16(2):10110.

94 Khedr EM, Farghaly WM, Amry S-D, Osman AA. Neural maturation of breastfed and formula-fed infants. Acta Paediatr. 2004 Jun; 93(6):734-8.

95 Walker A. Breast milk as the gold standard for protective nutrients. J Pediatr. 2010 Feb;156(2 Suppl):S3-7.

96 Herba CM, Roza S, Govaert P, Hofman A, Jaddoe V, Verhulst FC, et al. Breastfeeding and early brain development: the Generation R study. Matern Child Nutr. 2013 Jul;9(3): $332-49$.

97 Deoni SC, Dean DC, Piryatinsky I, O’Muircheartaigh J, Waskiewicz N, Lehman $\mathrm{K}$, et al. Breastfeeding and early white matter development: a cross-sectional study. NeuroImage. 2013 Nov;82:77-86.

98 Jing H, Gilchrist JM, Badger TM, Pivik RT. A longitudinal study of differences in electroencephalographic activity among breastfed, milk formula-fed, and soy formula-fed infants during the first year of life. Early Hum Dev. 2010 Feb;86(2):119-25.

99 Marshall PJ, Bar-Haim Y, Fox NA. Development of the EEG from 5 months to 4 years of age. Clin Neurophysiol. 2002 Aug;113(8): 1199-208.
100 Field T, Diego M, Hernandez-Reif M. Prenatal depression effects on the fetus and newborn: a review. Infant Behav Dev. 2006 Jul;29(3):445-55.

101 Raju TN. Breastfeeding is a dynamic biological process: not simply a meal at the breast. Breastfeed Med. 2011 Oct;6(5):257-9.

102 Šešo-Šimić Đ, Sedmak G, Hof P, Šimić G Recent advances in the neurobiology of attachment behavior. J Transl Neurosci. 2010 Jan 1;1(2):148-59.

103 Beijers R, Riksen-Walraven JM, de Weerth C. Cortisol regulation in 12-month-old human infants: associations with the infants' early history of breastfeeding and co-sleeping. Stress. 2013 May;16(3):267-77.

104 Ellingsen DM, Leknes S, Løseth G, Wessberg J, Olausson $\mathrm{H}$. The neurobiology shaping affective touch: expectation, motivation, and meaning in the multisensory context. Front Psychol. 2016 Jan 6;6:1986-16.

105 Fu Y, Selcuk E, Moore SR, Depue RA. Touch-induced face conditioning is mediated by genetic variation in opioid but not oxytocin receptors. Sci Rep. 2018 Dec;8(1): $1-10$.

106 Maletic V, Eramo A, Gwin K, Offord SJ, Duffy RA. The role of norepinephrine and its $\alpha$-adrenergic receptors in the pathophysiology and treatment of major depressive disorder and schizophrenia: a systematic review. Front Psychiatry. 2017 Mar 17;8:1-12.

107 Petersson M, Uvnäs-Moberg K, Erhardt S, Engberg G. Oxytocin increases locus coeruleus alpha 2-adrenoreceptor responsiveness in rats. Neurosci Lett. 1998 Oct 16;255(2): 115-8.

108 Thatcher RW, Walker RA, Giudice S. Human cerebral hemispheres develop at different rates and ages. Science. 1987 May 29; 236(4805):1110-3

109 Dennis CL, McQueen K. Does maternal postpartum depressive symptomatology influence infant feeding outcomes? Acta Paediatr. 2007 Apr;96(4):590-4. 